\title{
Association between cardiometabolic health and objectively-measured, free-living sleep parameters: a pilot study in a rural African setting
}

\author{
Ian Cook $^{\text {* }^{*} \text {, Matlawa Mohlabe }}{ }^{2}$ and Herbert Mabalane Makgopa ${ }^{2}$
}

\begin{abstract}
Objectives: To investigate the relationship between objectively-measured, free-living sleep quantity and quality, and cardiometabolic health, in a rural African setting in 139 adults ( $\geq 40$ years, female: $n=99$, male: $n=40$ ). Wristmounted, tri-axial accelerometry data was collected over 9 days. Measures of sleep quantity and quality, and physical activity were extracted from valid minute-by-minute data. Self-reported data included behavioural, health and socio-demographic variables. Biological data included body composition, resting blood pressure and fasting blood glucose, insulin and lipids. Logistic regression models were constructed with insulin resistance (IR) and cardiometabolic (CM) risk, as dependent variables, adjusting for socio-demographic, behavioural and biological factors.

Results: Nocturnal sleep time was longer in females $(p=0.054)$ and sleep quality was better in males $(p \leq 0.017)$. Few participants slept $>9 \mathrm{~h} /$ night (4-5\%), and $46-50 \%$ slept $<7 \mathrm{~h} /$ night. IR and CM risk was higher in females ( $p \leq$ 0.006). In adjusted models, sleep variables were independently associated with $\mathbb{I R}(p<0.05)$. Sleep quantity was nonlinearly associated with CM risk ( $p \leq 0.0398)$, and linearly associated with $I R(p \leq 0.0444)$. Sleep quality was linearly related with CM risk and IR ( $p \leq 0.0201)$. In several models, sleep quantity and sleep quality measures were concurrently and significantly associated with IR ( $p \leq 0.044)$.
\end{abstract}

Keywords: Insulin resistance, Metabolic syndrome, Anthropometry, Accelerometer, Actigraphy, Movement monitor, Measurement

\section{Introduction}

Sleep health is closely linked to metabolic health with several mechanisms linking poor sleep health to insulin resistance and the metabolic syndrome (Smiley et al. 2019). Although there is extensive literature from industrialised settings (Anothaisintawee et al. 2016), there is a paucity of data from African settings, especially freeliving, objective measures of sleep (Cole et al. 2017).

\footnotetext{
* Correspondence: ian.cook@ul.ac.za

'Physical Activity Epidemiology Laboratory (EDST), University of Limpopo (Turfloop Campus), PO Box X1106, Sovenga, Limpopo Province 0727, South Africa

Full list of author information is available at the end of the article
}

Within the South African context, self-reported long sleep duration is associated with poor cardiometabolic health in mainly urban settings (Rae et al. 2018; Rae et al. 2020). Given the lack of objectively-measured, freeliving sleep parameters in any South African setting, the objective of this study was to use wrist-actigraphy to investigate the association between sleep parameters and cardiometabolic health in a rural African setting during a cross-sectional survey, and thus extend the findings of self-report sleep duration and cardiometabolic health (Rae et al. 2018; Rae et al. 2020; Mashinya et al. 2018).

(c) The Author(s). 2021 Open Access This article is licensed under a Creative Commons Attribution 4.0 International License, which permits use, sharing, adaptation, distribution and reproduction in any medium or format, as long as you give appropriate credit to the original author(s) and the source, provide a link to the Creative Commons licence, and indicate if changes were made. The images or other third party material in this article are included in the article's Creative Commons licence, unless indicated otherwise in a credit line to the material. If material is not included in the article's Creative Commons licence and your intended use is not permitted by statutory regulation or exceeds the permitted use, you will need to obtain permission directly from the copyright holder. To view a copy of this licence, visit http://creativecommons.org/licenses/by/4.0/. 


\section{Main text \\ Methods \\ Dikgale health and demographic surveillance system site (DHDSS) sample}

A convenience sample of 167 adults was recruited from the DHDSS site (April 2016 - October 2017) (Alberts et al. 2015). These participants formed part of a larger study cohort ( $\geq 40$ years) (Mashinya et al. 2018; Ali et al. 2018). Trained field workers collected self-reported and measured data from participants by means of questionnaires translated to the local vernacular (Sepedi), anthropometry, oscillometric blood pressure measurement, ultrasound scans, and venipuncture (Mashinya et al. 2018; Ali et al. 2018). We calculated body mass index $\left(\mathrm{BMI}, \mathrm{kg} / \mathrm{m}^{2}\right.$ ), and Conicity Index (CI) (Valdez et al. 1993). Questionnaire data included behavioural, health and socio-demographic variables (Mashinya et al. 2018; Ali et al. 2018). Nine day, free-living, wrist-mounted accelerometry data was collected (Cook et al. 2020). The ultrasound scans were not considered for this analysis.

\section{Blood sample collection and analysis}

A registered nurse collected fasting blood samples. The samples were analysed centrally; procedures and calculations are described in detail elsewhere (Ali et al. 2018). The Homeostasis Model Assessment of Insulin Resistance (HOMA-IR) was calculated from fasting blood glucose and insulin (Matthews et al. 1985).

\section{Criteria for metabolic syndrome}

In accordance with the harmonized Joint Interim Statement (JIS) definition (Alberti et al. 2009), the presence of the Metabolic Syndrome (MetS) required three of the following components, with waist circumference not a prerequisite: elevated waist circumference (WC): females $\geq 92 \mathrm{~cm}$, males $\geq 86 \mathrm{~cm}$; elevated triglycerides (TG): $\geq 1.7$ $\mathrm{mmol} / \mathrm{l}$; reduced high-density lipoprotein cholesterol (HDL-C): men < $1.0 \mathrm{mmol} / \mathrm{l}$, women $<1.3 \mathrm{mmol} / \mathrm{l}$; elevated resting blood pressure $\geq 130 / 85 \mathrm{mmHg}$ or on hypertension treatment; and elevated fasting glucose (GC) $\geq 5.6 \mathrm{mmol} / \mathrm{l}$ or on diabetes treatment. For this study population-specific WC cut-points were chosen (Motala et al. 2011).

Using the five criteria from the JIS definition for MetS (JIS-MetS), we calculated sex-specific z-scores for HDLC, TG, GC, WC and MAP (Mean Arterial Pressure), which were summed to create a MetS z-Score (MetSz) (Lee et al. 2019).

\section{Accelerometer data collection and data reduction}

Participants wore a small, light-weight, wrist-worn triaxial accelerometer for 9 days (ActiGraph wGT3X-BT, Actigraph, LLC, Pensacola, FL, 2013) (Whitaker et al. 2018; Migueles et al. 2017; Slater et al. 2015; Full et al.
2018). The preparation, initialization, mounting, data processing and extraction of physical activity (PA) and sleep parameters are described in detail elsewhere (Cook et al. 2020).

Vector Magnitude (VM)- and Ambulation-defined PA variables were defined as counts/day and steps/day, respectively (Wennman et al. 2019). Sleep indices included Total Sleep Time (TST), Nocturnal Sleep Time (NST), Sleep Efficiency (SE), Wake After Sleep Onset (WASO), Activity Counts during sleep (AC), Sleep Fragmentation Index (SFI) and sleep variation across days (within-person total sleep time SD) (Ancoli-Israel et al. 2015; Chung et al. 2016; Ko and Lee 2018). Nocturnal periods were defined as 18 h01-05 h59. Sufficient sleep quantity and quality was defined as 7-9h (Hirshkowitz et al. 2015) and $\geq 85 \%$ (Fung et al. 2013), respectively.

\section{Statistical analysis}

Descriptive statistics comprised means (one standard deviation), medians (inter-quartile range), variances (maximum, minimum) and frequencies. Relationships between categorical variables were examined through Fisher's Exact Test. For continuous data, independent $t$ tests and Levene's test examined differences between sexes and risk groups. Where required a non-parametric test was employed. Bi-variate relationships were examined using linear regression.

Forced-entry binary logistic regression models were constructed to examine the relationship between MetS risk (low/high) according to the JIS definition (Alberti et al. 2009), and tertiles $(\mathrm{Q} 1=$ low $/ \mathrm{Q} 2 / \mathrm{Q} 3=$ high $)$ of sleep quantity and quality variables. Models were also constructed to examine the relationship between MetS risk and sleep quantity and quality categories (Hirshkowitz et al. 2015; Fung et al. 2013). Models were adjusted for socio-demographic (age, socio-economic status), behavioural (fruit and vegetable intake, sugar-sweetened beverages, tobacco and alcohol usage, physical activity) and biological (sex, HIV status) variables. CI was not included as an independent variable because WC formed part of the JIS risk definition.

Forced-entry ordinal logistic regression models were constructed to examine the relationship between HOMA-IR levels (tertiles), and tertiles of sleep quantity and quality variables. Additional models were constructed to examine the relationship between HOMA-IR tertiles and sleep quantity and quality categories (Hirshkowitz et al. 2015; Fung et al. 2013). Models were adjusted for socio-demographic (age, socio-economic status), behavioural (fruit and vegetable intake, sugarsweetened beverages, tobacco and alcohol usage, physical activity) and biological (sex, CI, HIV status) variables. 
All covariates were entered as quantiles. Regression coefficients were expressed as odds ratios (OR $\pm 95 \%$ confidence intervals).

Goodness of fit criteria included Akaike's Information Criteria (AIC) (all models), pseudo $\mathrm{R}^{2}$ (all models), Hosmer-Lemeshow test (Binary logistic) and Area Under the Curve (AUC) (Binary Logistic). Multicollinearity was assessed using Variance Inflation Factors (VIF) and Tolerance.

Post-hoc contrasts were run for binary and ordinal logistic regression models to test for linear and non-linear trends, and pairwise comparison of groups (Bonferroni correction).

Data were analysed using appropriate statistical software (Stata/SE for Windows: Release 15.1. College Station, TX: StataCorp LP, 2020). Significance for all inferential statistics was set at $p<0.05$.

\section{Results}

Of the 167 raw accelerometry data files, 157 had valid data for at least one weekday and one weekend day. Once combined with the questionnaire and biological data, 139 participants had complete data.

Females consumed more sugar-sweetened beverages (SSB), experienced poorer sleep quality, and were more physically active and insulin resistant (Table $1, p \leq 0.07$ ). Males were leaner, used more alcohol and tobacco products and experienced lower people-to-bedroom density $(p \leq 0.040)$. The JIS high risk group had a higher socioeconomic status (SES), a lower HIV+ prevalence, were mostly obese, more insulin resistant, and likely to be in a partnered relationship (Table 1, $p \leq 0.040$ ).

In bi-variate analysis, sex, SES, CI, WC, BMI, SSB and WASO were significantly associated with HOMA-IR $(p \leq 0.05)$. SES, BMI, CI, WC and HIV status were significantly related to MetSz $(p \leq 0.027)$.

All models were significant (Fig. 1a-f, Fig. 2a-f, see Additional file 1 for Fig. S1A-B, $p \leq 0.00191$ ) and there was no evidence of multicollinearity (VIF $<1.5$, Tolerance $>0.75$ ). The binary logistic regression models (Fig. 1a-c, Fig. 2a-c, Additional file 1: Fig. S1A) showed good fit (Hosmer-Lemeshow: $p \geq 0.2223$; AUC: 0.808-0.877). The full models (OR $\pm 95 \%$ confidence intervals) for Fig. 1a-f, Fig. 2a-f and Additional file 1: Fig. S1A-B are available in Additional file 2.

In binary logistic regression models (Fig. 1a-c, Additional file 1: Fig. S1A) males had significantly lower odds for JIS-MetS high risk (OR $\approx 0.15, p \leq 0.006)$, and similarly for HIV+ status in the NST and SFI models $(\mathrm{OR}=0.25, p \leq 0.0035)$. Once adjusted for adiposity $(\mathrm{CI})$, sex was no longer a significant factor $(p \geq 0.097)$. However, HIV+ status remained a significant factor in most models (OR $\approx 0.26, p \leq 0.048$ ) (Fig. 1a-c).
There was a significant non-linear trend (U-shaped) between JIS-MetS risk and NST $(p=0.0196)$ (Fig. 1a), such that for NST Q2, there was 80\% less likelihood of being at high risk for JIS-MetS. There was also a significant difference in frequencies between NST Q1 and Q2 $(p=0.021)$ (Fig. 1a).

There was a significant positive, linear trend between JIS-MetS risk and SFI ( $p=0.0001)$ (Fig. 1c). The odds of high risk JIS-MetS for SFI Q3 was high $(\mathrm{OR}=69.81$, 8.44; 577.63), however the confidence intervals were wide. There were significant differences between the frequencies for Q3 versus Q1 and Q2 $(p<0.001)$ (Fig. 1c).

Being a current user of both alcohol and tobacco products carried a significant higher odds for JIS-MetS high risk in the NST (OR $=4.51, p=0.034)$ and SFI $(\mathrm{OR}=$ 6.55, $p=0.023$ ) models (Fig. 1a-c).

In ordinal logistic regression models (Fig. 1d-f, Additional file 1: Fig. S1B), being in the VM Q2 level, significantly decreased the odds of being in HOMA-IR Q3 by a factor of $\approx 0.31(p \leq 0.039)$. In contrast, for both CI Q2 and Q3 levels, the odds of being in HOMA-IR Q3 were significantly increased; Q2 OR 2.9 and Q3 $\mathrm{OR} \approx 3.8$, respectively $(p \leq 0.045)$ (Fig. $1 \mathrm{~d}-\mathrm{f}$, Additional file 1: Fig. S1B).

There was a significant linear trend between TST and HOMA-IR levels $(p=0.0444)$ (Fig. 1d). Compared to TST Q1 and Q2, being in TST Q3 (longest sleep time) increased the odds of being in the highest HOMA-IR level (Q3) by a factor of $2.84(p=0.044)$ (Fig. 1d).

There were significant linear relationships between SE and WASO, and HOMA-IR ( $p \leq 0.007)$ (Fig. 1e). A high SE (Q3) was 85\% less likely to result in HOMA-IR Q3 $(p=0.001)$. Being in WASO Q2 and Q3 significantly increased the odds for HOMA-IR Q3; OR = 3.17 and OR = 6.75 , respectively $(p \leq 0.019)$.

SFI and AC were significantly associated with increasing HOMA-IR levels $(p \leq 0.0201)$ (Fig. 1f). Being in SFI Q2 and Q3 significantly increased the odds for HOMAIR Q3, OR $=4.67(p=0.004)$ and $\mathrm{OR}=10.91(p<0.001)$, respectively. For AC Q3 the odds of being in the HOMA-IR Q3 was $3.01(p=0.020)$ (Fig. 1f).

In the TST, NST, SE, WASO and AC models (Fig. 1de), being in SSB Q2 and Q3, increased the likelihood for being in HOMA-IR Q3 by a factor of 3.51 to 4.77 , but did not reach statistical significance ( $p=0.068$ to $p=0.098$ ).

Sleep quantity and quality measures featured concurrently and significantly in the TST, SE and SFI models (Fig. 1d-f) $(p \leq 0.044)$.

Expressing sleep quantity and quality parameters in terms of sleep health guidelines, we found significant non-linear associations with JIS-MetS risk $(p \leq 0.0308)$ (Fig. 2a-f). The relationship between sleep categories for both TST and NST, and JIS-MetS risk were U-shaped, the nadir at 7-9 $\mathrm{h}$ of sleep (Fig. 2a-b). 
Table 1 Descriptive statistics of demographic, behavioural and biological characteristics by sex and cardio-metabolic risk

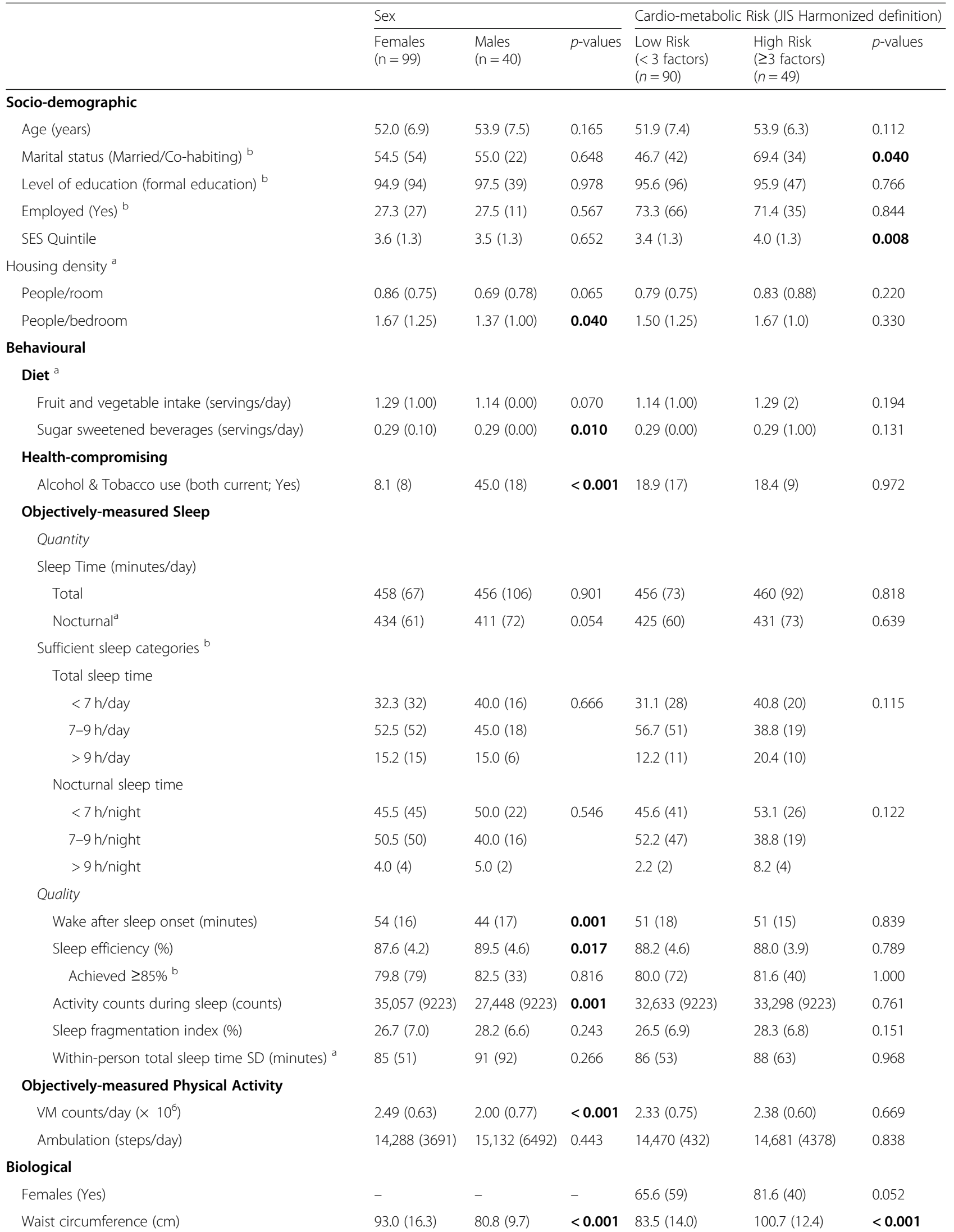


Table 1 Descriptive statistics of demographic, behavioural and biological characteristics by sex and cardio-metabolic risk (Continued)

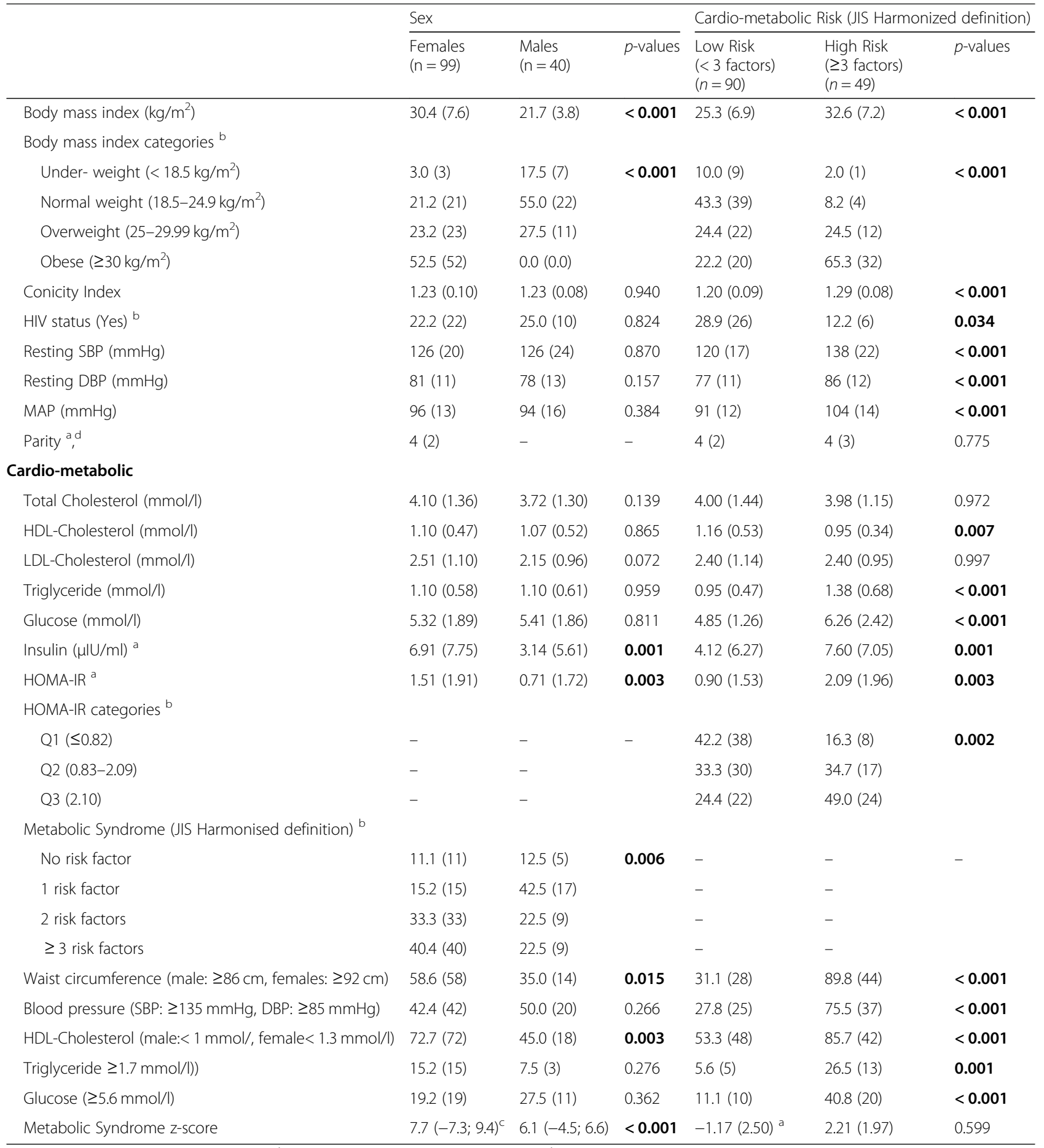

Data reported as mean (SD), ${ }^{a}$ median (IQR), ${ }^{\mathrm{b}} \%(\mathrm{n})$ or ${ }^{\mathrm{c}}$ variance (minimum; maximum); ${ }^{\mathrm{d}}$ low risk: $n=59$, high risk: $\mathrm{n}=40 ;$ DBP Diastolic Blood Pressure, $H O M A-I R$ Homeostasis Model Assessment of Insulin Resistance, HIV Human Immunodeficiency Virus, JIS Joint Interim Statement, MAP Mean Arterial Pressure $=2 / 3$ DBP $+1 / 3$ 


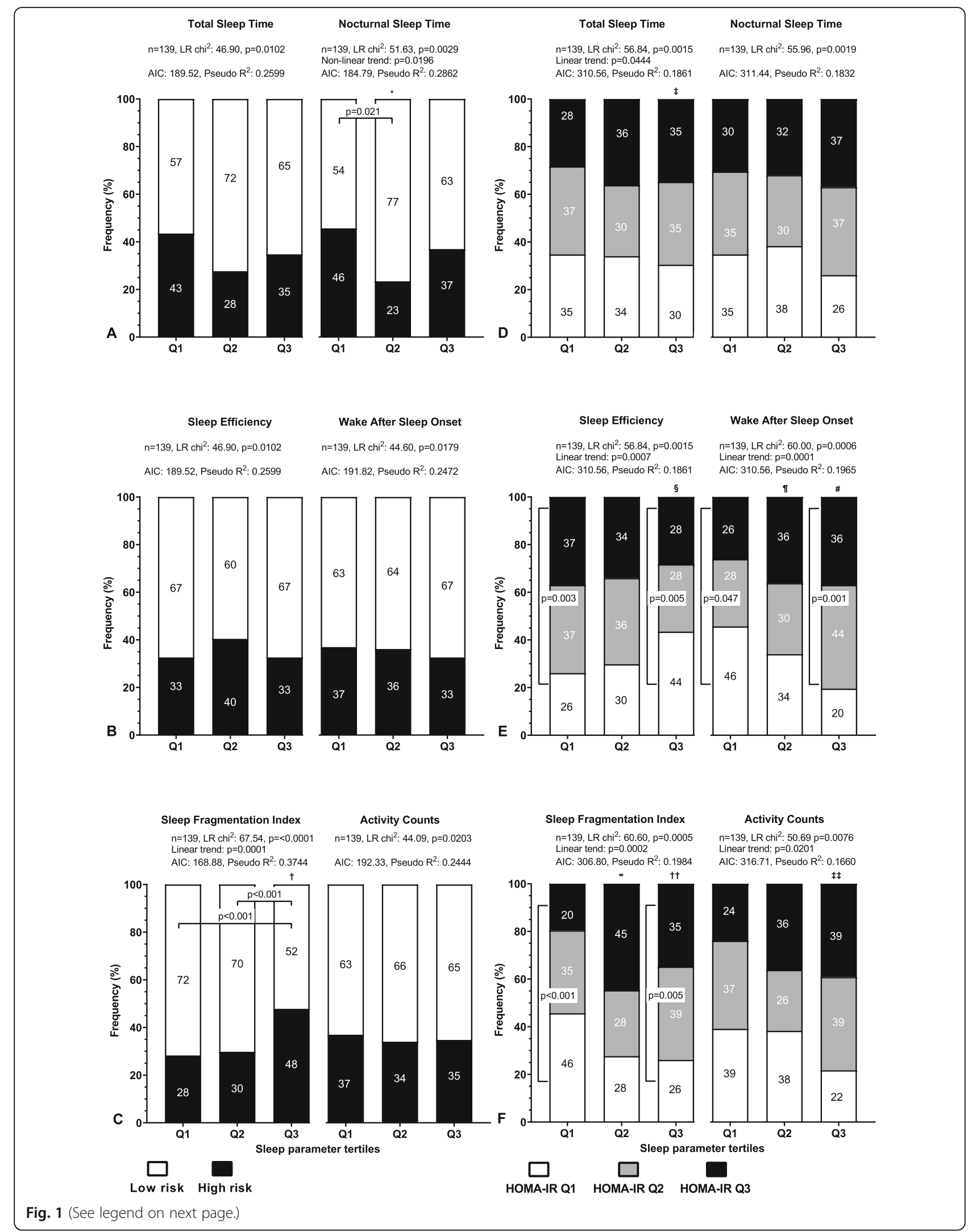




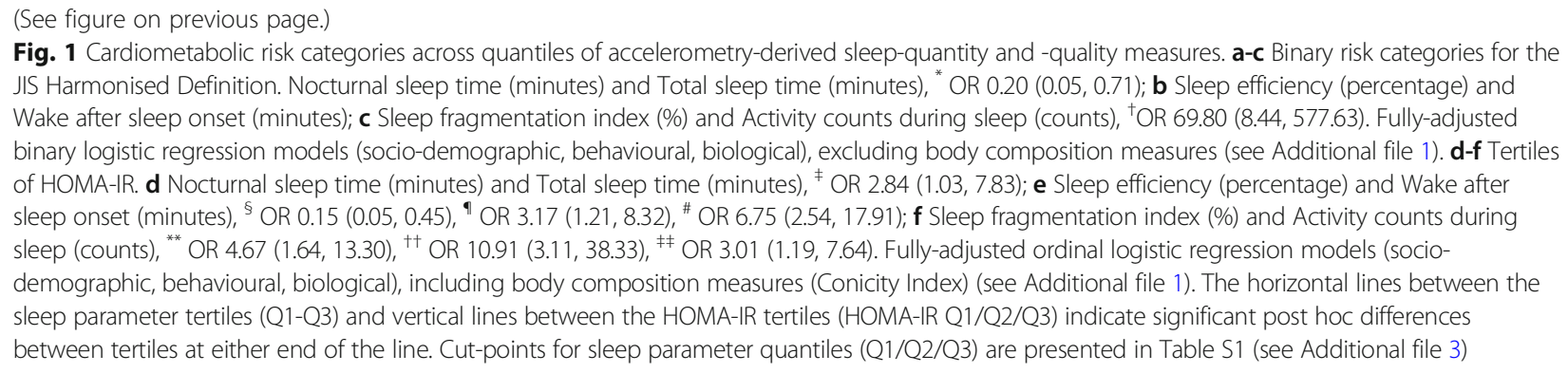

In binary logistic regression models (Fig. 2a-c) males had significantly lower odds for JIS-MetS high risk $(\mathrm{OR} \approx 0.14, p=0.004)$, and similarly for $\mathrm{HIV}+(\mathrm{OR} \approx 0.26$, $p \leq 0.0034)$. Once adjusted for adiposity (CI), sex was no longer a significant factor $(p \geq 0.121)$. However, HIV+ status remained a significant factor in most models $(\mathrm{OR} \approx 0.23, p \leq 0.045)$ (Fig. 2a-c).

In ordinal logistic regression models (Fig. 2d-f) physical activity (VM Q2) significantly reduced the odds of being in HOMA-IR Q3 by a factor of $\approx 0.26(p \leq 0.010)$. For both CI Q2 and Q3 levels, the odds of being in HOMA-IR Q3 were significantly increased (Q2 $\mathrm{OR} \approx 3.16$, Q3 $\mathrm{OR} \approx 3.89, p \leq 0.022$ ). For $\mathrm{SE} \geq 85 \%$, there was a 76\% lower likelihood of being in HOMA-IR Q3 $(p=0.010)$. Significantly more participants were classified as low HOMA-IR (Q1) in the SE $\geq 85 \%$ category (Fig. 2d).

In the TST, NST and SE models (Fig. 2d and f), being in SSB Q2, increased the odds for being in HOMA-IR Q3 by a factor of 3.24 to 3.65 , but did not reach statistical significance $(p=0.074)$.

\section{Discussion}

This analysis is novel in that, as far as the authors are aware, this is the first free-living, actigraphy-measured sleep and cardiometabolic health study from a rural South African setting.

The main findings of this analysis were first that sleep quality and quantity measures were independently associated with HOMA-IR, and to a lesser extent JIS-MetS. Second, we found linear and non-linear (U-shaped) relationships between categories of sleep quantity and cardiometabolic risk. Third, except for sleep variability, all sleep quality measures were consistently associated with HOMA-IR.

The level of JIS-defined MetS within rural sub-Sahara African settings is $12.0 \%$ (95\%CI: 4.0; 23.4) which is substantially lower than in our sample (35.3\%) and is likely due to variations in levels of central adiposity, differing stages of the epidemiological transition and variations in the implementation of preventative programmes (Jaspers Faijer-Westerink et al. 2020). However, the mean prevalence reported in a rural South African setting for the $\geq 45$ year age groups (males: $17.9 \%$; females: $42.2 \%$ ) (Motala et al. 2011) is similar to our results (males: 22.5\%; females: $40.4 \%$ ).

Our findings are in agreement with the linear relationship between sleep duration and HOMA-IR in black, urban women, although we found far more women had short sleep time compared with self-report measures (Rae et al. 2018). A recent study from the METS group found long sleep duration in a black, urban South African sample (Rae et al. 2020). The accuracy with which sleep is self-reported, and how sleep questions are interpreted across different South African populations is unknown (Rae et al. 2018; Rae et al. 2020).

Unadjusted sleep quantity did not differ significantly across the sexes, which is in contrast to self-report measures (Rae et al. 2018). Sleep quality was poorer in females, although sex did not reach significance in most HOMA-IR models. Poor sleep quality in females is likely due to environmental and social determinants (Cook et al. 2020).

Some have speculated that poor sleep quality might be underpinning the long self-reported sleep durations in South African settings and hence the poorer cardiometabolic health associated with long sleep (Rae et al. 2018; Rae et al. 2020). Our results suggest that fragmented, poor sleep quality, independent of sleep duration might be more important than sleep duration. In young adolescents objectively-measured sleep quality, independent of sleep duration, was associated with cardiometabolic risk, such that increasing sleep duration and better sleep quality were associated with better cardiometabolic health (Feliciano et al. 2018). Future analyses need to explore the effect of the interaction between sleep quantity and quality, and cardiometabolic health (Lu et al. 2020).

Higher sleep variability for objectively-measured sleep quantity and quality measures has been shown to be associated with less favourable cardiometabolic health (Baron et al. 2017). In contrast, we found no association between sleep variability in total sleep time and cardiometabolic health. Median sleep variability was $22 \mathrm{~min}$ higher in our rural study compared with an 


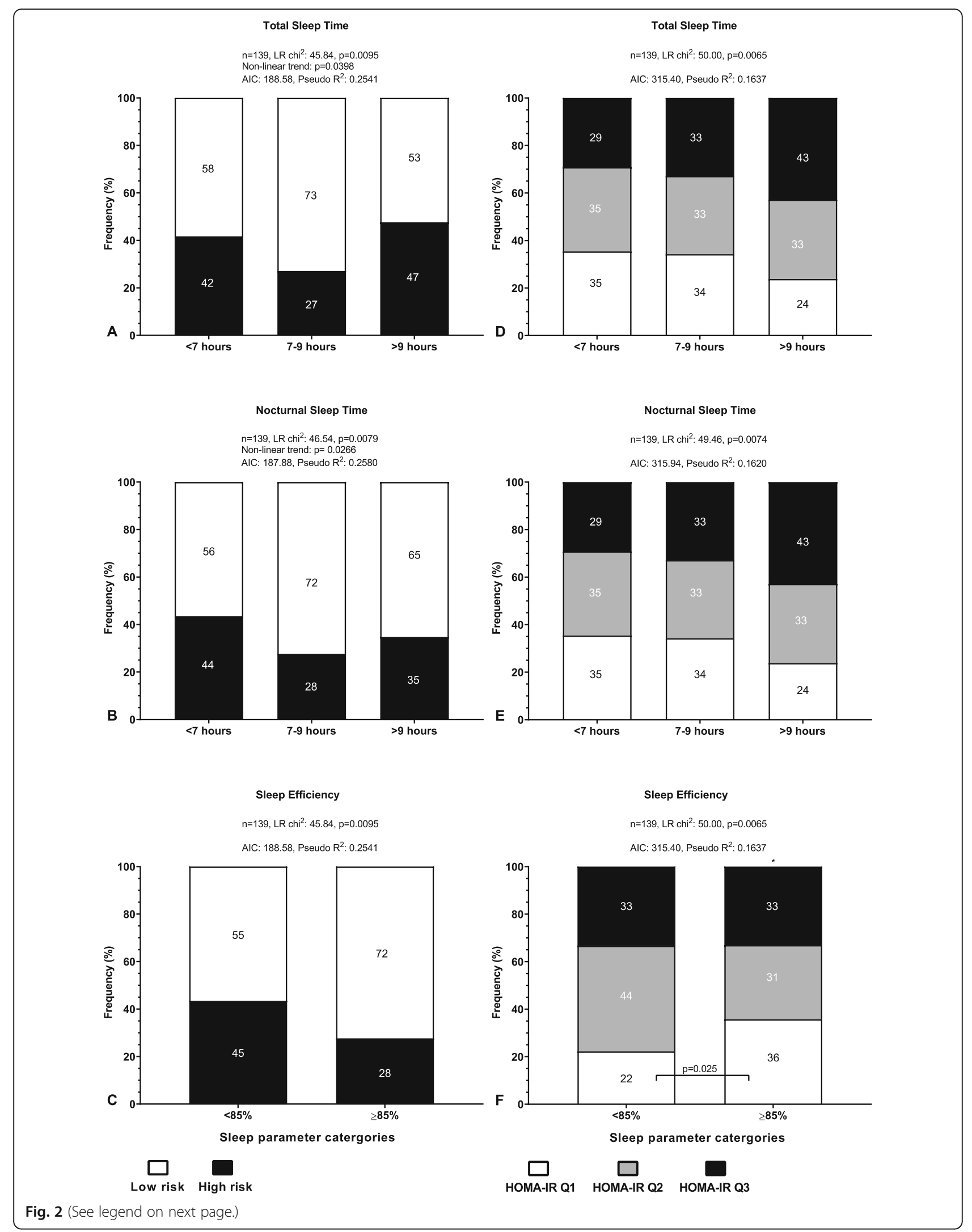


(See figure on previous page.)

Fig. 2 Cardiometabolic risk categories across categories of accelerometry-derived sleep-quantity and -quality measures. a-c Binary risk categories for the JIS Harmonised Definition. Total sleep time (minutes); b Nocturnal sleep time (minutes); c Sleep efficiency (percentage). Fully-adjusted binary logistic regression models (socio-demographic, behavioural, biological), excluding body composition measures (see Additional file 1). $\mathbf{d}-\mathbf{f}$ Tertiles of HOMA-IR. $\mathbf{d}$ Total sleep time (minutes); e Nocturnal sleep time (minutes); f Sleep efficiency (percentage), ${ }^{*}$ OR 0.24 (0.08, 0.71$)$. Fullyadjusted ordinal logistic regression models (socio-demographic, behavioural, biological), including body composition measures (Conicity Index) (see Additional file 1). The horizontal lines between the sleep parameter tertiles (Q1-Q3) indicate significant post hoc differences between tertiles at either end of the line

industrialised, urban, adult sample; $87 \mathrm{~min}$ vs. $65 \mathrm{~min}$, respectively (DeSantis et al. 2019).

Future investigations into the sleep and cardiometabolic health of this rural group will require the construction of a composite sleep health score as opposed to relying solely on individual sleep dimensions (DeSantis et al. 2019).

In contrast with some self-reported PA studies (Rae et al. 2018), we found PA volume to be significantly and independently related to HOMA-IR, but not associated with JIS-MetS risk as in other self-report studies (Rae et al. 2020). Interestingly, other lifestyle factors such as concurrent alcohol and tobacco use, and the consumption of SSB were independently associated with poor cardiometabolic health. The concurrent use of alcohol and tobacco is associated with poorer cardiometabolic health through dyslipidemia and abdominal obesity (Slagter et al. 2014). Although the association with SSB did not quite reach statistical significance, there is evidence that the consumption of SSB is linked with poor cardiometabolic health through insulin resistance, visceral adiposity, dyslipidemia and inflammation, even within rural environments (Vorster et al. 2014).

\section{Conclusion}

We found objectively-measured sleep quality indices were significantly associated with HOMA-IR in a rural South African sample. Future research in this population should include composite sleep health indices, and detailed data for environmental and social factors which impact sleep health. This study suggests that poor sleep quality, independent of sleep duration, may be an important risk factor for the development insulin resistance. Identifying and addressing factors which influence sleep quality should be considered as integral to strategies and interventions aimed at addressing cardiometabolic health in this rural population.

\section{Limitations}

Due to the small sample size and cross-sectional, convenience sampling in this study, the results cannot be readily generalized, nor can causality be shown.

\section{Supplementary Information}

The online version contains supplementary material available at https://doi. org/10.1186/s41606-020-00054-y.

Additional file 1. Cardiometabolic risk categories across categories of accelerometry-derived sleep variability. A: Binary risk categories for the JIS Harmonised Definition. Total sleep time SD (minutes). Fullyadjusted binary logistic regression model (socio-demographic, behavioural, biological), excluding body composition measures (see Additional file 1). B: Tertiles of HOMA-IR. Total sleep time SD (minutes). Fullyadjusted ordinal logistic regression model (socio-demographic, behavioural, biological), including body composition measures (Conicity Index) (see Additional file 1). Cut-points for sleep parameter quantiles (Q1/Q2/ Q3) are presented in Additional file 3: Table S1 (see Additional file 3)

Additional file 2. Models. Full logistic regression models for Fig. 1a-f, Fig. 2a-f and Additional file 1: Fig. S1A-B.

Additional file 3: Table S1. Cut-points for quantiles of sleep parameters.

\section{Abbreviations}

BMI: Body mass index; Cl: Conicity Index; DHDSS: Dikgale Health and Demographic Surveillance System; GC: Glucose; HDL-C: High-density Lipoprotein Cholesterol; HIV: Human Immunodeficiency Virus; HOMA-

IR: Homeostasis Model Assessment of Insulin Resistance; JIS: Joint Interim Statement; LDL-C: Low-density Lipoprotein Cholesterol; MAP: Mean Arterial Pressure; MetS: Metabolic Syndrome; MetSz: Metabolic Syndrome z-score; NST: Nocturnal Sleep Time; PA: Physical Activity; SE: Sleep Efficiency; SES: Socio-Economic Status; SFI: Sleep Fragmentation Index; SSB: Sugar sweetened beverages; TC: Total Cholesterol; TG: Triglyceride; TST: Total Sleep Time; VM: Vector Magnitude; WASO: Wake After Sleep Onset; WC: Waist circumference

\section{Acknowledgements}

The authors would like to thank the communities, participants and field workers of the Dikgale Demographic and Health Surveillance System Site.

\section{Authors' contributions}

IC was the principal investigator of the accelerometer data on which this manuscript is based, who initiated the research and obtained funding, cowrote and edited the research proposal, supervised the data entry, analyzed the data and wrote the first draft manuscript. MM co-wrote and edited the research proposal, collected field data, supervised the field work, performed the data entry, commented on the manuscript. HMM commented on- and contributed to the manuscript. The authors read and approved the final manuscript.

\section{Funding}

The AWI-Gen Collaborative Centre is funded by the National Human Genome Research Institute (NHGRI), Office of the Director (OD), Eunice Kennedy Shriver National Institute Of Child Health \& Human Development (NICHD), the National Institute of Environmental Health Sciences (NIEHS), the Office of AIDS research (OAR) and the National Institute of Diabetes and Digestive and Kidney Diseases (NIDDK), of the National Institutes of Health $(\mathrm{NIH})$ under award number U54HG006938 and its supplements, as part of the H3Africa Consortium. Additional funding was leveraged from the Department of Science and Technology, South Africa, award number DST/CON 0056/2014, and from the African Partnership for Chronic Disease Research (APCDR). The 
accelerometry section of this study was funded by the Competitive Programme for Rated Researchers, National Research Foundation, South Africa, award number CPRR14080687621.

\section{Availability of data and materials}

The dataset analysed during the current study is available from the corresponding author on reasonable request.

\section{Ethics approval and consent to participate}

Ethics approval for the AWI-Gen Phase 1 survey data and the accelerometer data collection was obtained from the Medunsa Research and Ethics Committee of the University of Limpopo (MREC/HS/195/2014:CR). The participants recruited into the original studies were informed about the study objectives, expected outcomes, benefits and the risks associated with it. Written informed consent was obtained from the participants prior to interviews and measurements.

\section{Consent for publication}

Not applicable.

\section{Competing interests}

The authors declare no competing interests.

\section{Author details}

${ }^{1}$ Physical Activity Epidemiology Laboratory (EDST), University of Limpopo (Turfloop Campus), PO Box X1106, Sovenga, Limpopo Province 0727, South Africa. ${ }^{2}$ Department of Pathology and Medical Sciences, University of Limpopo (Turfloop Campus), Sovenga, Limpopo Province, South Africa.

\section{Received: 1 August 2020 Accepted: 24 November 2020}

Published online: 07 January 2021

\section{References}

Alberti KG, Eckel RH, Grundy SM, Zimmet PZ, Cleeman JI, Donato KA, et al. Harmonizing the metabolic syndrome: a joint interim statement of the international diabetes federation task force on epidemiology and prevention; National Heart, Lung, and Blood Institute; American Heart Association; world heart federation; international atherosclerosis society; and International Association for the Study of obesity. Circulation. 2009;120:1640-5.

Alberts M, Dikotope SA, Choma SR, Masemola ML, Modjadji SE, Mashinya F, et al. Health \& Demographic Surveillance System Profile: the Dikgale health and demographic surveillance system. Int J Epidemiol. 2015;44:1565-71.

Ali SA, Soo C, Agongo G, Alberts M, Amenga-Etego L, Boua RP, et al. Genomic and environmental risk factors for cardiometabolic diseases in Africa: methods used for phase 1 of the AWl-gen population cross-sectional study. Glob Health Action. 2018;11:1507133.

Ancoli-Israel S, Martin JL, Blackwell T, Buenaver L, Liu L, Meltzer LJ, et al. The SBSM guide to actigraphy monitoring: clinical and research applications. Behav Sleep Med. 2015;13:S4-S38

Anothaisintawee T, Reutrakul S, Van Cauter E, Thakkinstian A. Sleep disturbances compared to traditional risk factors for diabetes development: systematic review and meta-analysis. Sleep Med Rev. 2016;30:11-24.

Baron KG, Reid KJ, Malkani RG, Kang J, Zee PC. Sleep variability among older adults with insomnia: associations with sleep quality and cardiometabolic disease risk. Behav Sleep Med. 2017;15:144-57.

Chung S, Youn S, Lee C, Jo M-W, Park J, Jo SW, et al. Environmental noise and sleep disturbance: night-to-night variability of sleep/wake pattern. Sleep Med Res. 2016;7:78-81.

Cole HV, Owusu-Dabo E, Iwelunmor J, Newsome V, Meeks K, Agyemang C, et al. Sleep duration is associated with increased risk for cardiovascular outcomes: a pilot study in a sample of community dwelling adults in Ghana. Sleep Med. 2017:34:118-25.

Cook I, Mohlabe M, Alberts M. Descriptive epidemiology of objectively-measured, free-living sleep parameters in a rural African setting. BMC Res Notes. 2020; 13:310.

DeSantis AS, Dubowitz T, Ghosh-Dastidar B, Hunter GP, Buman M, Buysse DJ, et al. A preliminary study of a composite sleep health score: associations with psychological distress, body mass index, and physical functioning in a low-income African American community. Sleep Health. 2019:5:514-20.
Feliciano EMC, Quaante M, Rifas-Shiman SL, Redline S, Oken E, Taveras EM. Objective sleep characteristics and cardiometabolic health in young adolescents. Pediatrics. 2018;142:e20174085.

Full KM, Kerr J, Grandner MA, Malhotra A, Moran K, Godoble S, et al. Validation of a physical activity accelerometer device worn on the hip and wrist against polysomnography. Sleep Health. 2018;4:209-16.

Fung MM, Peters K, Ancoli-Israel S, Redline S, Stone KL, Barrett-Connor E. Total sleep time and other sleep characteristics measured by actigraphy do not predict incident hypertension in a cohort of community-dwelling older men. J Clin Sleep Med. 2013;9:585-91.

Hirshkowitz M, Whiton K, Albert SM, Alessi C, Bruni O, DonCarlos L, et al. National Sleep Foundation's sleep time duration recommendations: methodology and results summary. Sleep Health. 2015;1:40-3.

Jaspers Faijer-Westerink H, Kengne AP, Meeks KAC, Agyemang C. Prevalence of metabolic syndrome in sub-Saharan Africa: a systematic review and metaanalysis. Nutr Metab Cardiovasc Dis. 2020;30:547-65.

Ko Y, Lee J-Y. Effects of feet warming using bed socks on sleep quality and thermoregulatory responses in a cool environment. J Physiol Anthropol. 2018:37:13.

Lee JC, Westgate K, Boit MK, Mwaniki DL, Kiplamai FK, Friis H, et al. Physical activity energy expenditure and cardiometabolic health in three rural Kenyan populations. Am J Hum Biol. 2019;31:e23199-e.

Lu K, Zhao Y, Chen J, Hu D, Xiao H. Interactive association of sleep duration and sleep quality with the prevalence of metabolic syndrome in adult Chinese males. Exp Ther Med. 2020;19:841-8.

Mashinya F, Alberts M, Cook I, Ntuli S. Determinants of body mass index by gender in the Dikgale health and demographic surveillance system site, South Africa. Glob Health Action. 2018;11:1537613.

Matthews DR, Hosker JP, Rudenski AS, Naylor BA, Treacher DF, Turner RC. Homeostasis model assessment: insulin resistance and beta-cell function from fasting plasma glucose and insulin concentrations in man. Diabetologia. 1985;28:412-9.

Migueles JH, Cadenas-Sanchez C, Ekelund U, Delisle Nyström C, Mora-Gonzalez J, Löf $M$, et al. Accelerometer data collection and processing criteria to assess physical activity and other outcomes: a systematic review and practical considerations. Sports Med. 2017;47:1821-45.

Motala AA, Esterhuizen T, Pirie FJ, Omar MA. The prevalence of metabolic syndrome and determination of the optimal waist circumference cutoff points in a rural south African community. Diabetes Care. 2011;34:1032-7.

Rae DE, Dugas L, Roden L, Lambert EV, Bovet P, Plange-Rhule J, et al. Associations between self-reported sleep duration and cardiometabolic risk factors in young African-origin adults from the five-country modeling the epidemiologic transition study (METS). Sleep Health. 2020; 6:469-77.

Rae DE, Pienaar PR, Henst RHP, Roden LC, Goedecke JH. Associations between long self-reported sleep, obesity and insulin resistance in a cohort of premenopausal black and white south African women. Sleep Health. 2018;4: 558-64.

Slagter SN, van Vliet-Ostaptchouk JV, Vonk JM, Boezen HM, Dullaart RPF, Kobold ACM, et al. Combined effects of smoking and alcohol on metabolic syndrome: the LifeLines cohort study. PLoS One. 2014;9:e96406.

Slater JA, Botsis T, Walsh J, King S, Straker LM, Eastwood PR. Assessing sleep using hip and wrist actigraphy. Sleep Biol Rhythms. 2015;13:172-80.

Smiley A, Wolter S, Nissan D. Mechanisms of Association of Sleep and Metabolic Syndrome. J Med Clin Res Rev. 2019;3:1-9.

Valdez R, Seidell JC, Ahn Yl, Weiss KM. A new index of abdominal adiposity as an indicator of risk for cardiovascular disease. A cross-population study. Int J Obes Relat Metab Disord. 1993;17:77-82.

Vorster HH, Kruger A, Wentzel-Viljoen E, Kruger HS, Margetts BM. Added sugar intake in South Africa: findings from the adult prospective urban and rural epidemiology cohort study. Am J Clin Nutr. 2014;99:1479-86.

Wennman H, Pietilä A, Rissanen H, Valkeinen H, Partonen T, Mäki-Opas T, et al. Gender, age and socioeconomic variation in 24-hour physical activity by wrist-worn accelerometers: the FinHealth 2017 survey. Sci Rep. 2019;9:6534.

Whitaker KM, Pettee Gabriel K, Jacobs DR Jr, Sidney S, Sternfeld B. Comparison of two generations of ActiGraph accelerometers: the CARDIA study. Med Sci Sports Exerc. 2018;50:1333-40.

\section{Publisher's Note}

Springer Nature remains neutral with regard to jurisdictional claims in published maps and institutional affiliations. 